\title{
Amino acid starvation accelerates replication of Ibaraki virus
}

Keiko Onishi, ${ }^{a}$ Shusaku Shibutani, ${ }^{a}$ Nanami Goto, Yuki Maeda, and Hiroyuki Iwata*

Laboratory of Veterinary Hygiene, Joint Faculty of Veterinary Medicine, Yamaguchi University, 1677-1 Yoshida, Yamaguchi 753-8515, Japan

aThese authors contributed equally to this work.

${ }^{*}$ Corresponding author:

E-mail address: hiwata@yamaguchi-u.ac.jp (H. Iwata)

Key words: Ibaraki virus; mTOR; mTORC1; amino acid; endosome 


\section{ABSTRACT}

Ibaraki virus (IBAV) is a strain of epizootic hemorrhagic disease virus 2 that belongs to the genus Orbivirus of the family Reoviridae. IBAV replication is suppressed by the inhibition of autophagy, and since mechanistic target of rapamycin complex 1 (mTORC1) is a key regulator of autophagy, we examined if mTORC1 inhibition by amino acid starvation or mTOR inhibitors (Torin 1 and rapamycin) affects IBAV replication. We found that IBAV replication is significantly enhanced after amino acid starvation of host cells, but not after treatment with mTOR inhibitors, during early stages of viral infection (0-1 hpi). Notably, inhibition of mTORC1 by amino acid starvation was reversible and thus restricted to $0-1 \mathrm{hpi}$, whereas mTOR inhibitors sustainably suppressed mTORC1 even after the 1-h treatment, suggesting that mTORC1 suppression itself does not affect IBAV replication. To investigate the mechanism of enhanced IBAV replication by amino acid starvation, we examined the endocytic pathway, since IBAV utilizes acidification of endosomes as a trigger for viral replication. Accordingly, we found that amino acid starvation, but not mTOR inhibitors, strongly induced acidification of endosomes/lysosomes and that inhibition of endosomal acidification by bafilomycin A1 effectively blocked enhancement of IBAV replication. Altogether, the inactivation of mTORC 1 by amino acid starvation during early stages of infection enhances acidification of endosomes, which in turn enhances IBAV replication. 


\section{Introduction}

Orbiviruses belong to genus Orbivirus of the Reoviridae family and are transmitted by arthropod vectors. Orbiviruses contain many viruses, including bluetongue virus (BTV), African horse sickness virus (AHSV), and epizootic hemorrhagic disease virus (EHDV), that result in severe economic losses to the livestock industry (Gould and Hyatt, 1994). Ibaraki virus (IBAV) is a strain of EHDV-2, known as the causative agent of Ibaraki disease that is characterized by swallowing difficulties in cattle (Inaba, 1975). Although the spread of Ibaraki disease can be effectively prevented by vaccination, the recent emergence of IBAV in non-vaccinated cattle and the circulation of other EHDVs in southern Japan indicates the potential danger of new outbreaks (Hirashima et al., 2015). Despite the importance in understanding the mechanisms of IBAV infection, little is known about how IBAV infects host cells and how it expresses virulence. We have recently investigated the mechanism of IBAV entry into host mammalian cells and found that IBAV invades host cells by exploiting the endocytic pathway, similar to a related virus, BTV (Tsuruta et al., 2016). Furthermore, we found that acidification of the IBAVcontaining endosome acts as a trigger for IBAV infection, possibly by inducing structural changes in the outer capsid of IBAV (Tsuruta et al., 2016). Therefore, IBAV exploits host cell endocytosis, an important membrane trafficking process that contributes to cellular homeostasis.

In this study, we attempted to investigate the interaction between IBAV and autophagy, another membrane trafficking process. Autophagy is a cellular catabolic process that is widely conserved among eukaryotes from yeast to humans (Mizushima and Komatsu, 2011). Autophagy is induced by nutrient (especially amino acid) starvation and degrades macromolecules, such as cytoplasmic proteins, through their engulfment into a double-membrane vesicle called the autophagosome. The degradation products generated by autophagosome-lysosome fusion (e.g., amino acids generated by the digestion of proteins) will be recycled as building blocks for biosynthesis. Furthermore, autophagy is thought to maintain cellular homeostasis by eliminating unwanted intracellular molecules and structures, such as abnormal proteins, damaged organelles, and invasive pathogenic microorganisms (Mizushima and Komatsu, 2011). Autophagy is mainly regulated by mechanistic target of rapamycin complex 1 (mTORC1), a major kinase signaling complex that governs cellular metabolism (Mizushima et al., 2011). Under nutrient-rich conditions, mTORC1 is activated by amino acids and growth factors, and activated mTORC1 strongly inhibits autophagy through the inhibitory 
phosphorylation of the autophagy proteins, Atg13 and ULK1. In contrast, under starved conditions mTORC1 is inactivated, leading to the induction of autophagy.

The relationship between viral infection and autophagy has been studied in various viruses (Dreux and Chisari, 2010; Jackson, 2015). Some viruses inhibit autophagy to escape from autophagy-mediated destruction of virus particles, whereas others exploit autophagy to promote virus replication. For the Reoviridae family, it has been reported that autophagy is induced by viral infection and is exploited for efficient viral replication in avian reovirus (Chi et al., 2013; Meng et al., 2012), BTV (Gu et al., 2012; Lv et al., 2015), and IBAV (Shai et al., 2013). For IBAV, it was shown that pharmacological and genetic inhibition of autophagy results in the suppression of IBAV replication, suggesting that autophagy is required for optimal viral replication (Shai et al., 2013). Although the cause-effect relationship between autophagy inhibition and viral suppression has been demonstrated, the molecular mechanism behind this relationship remains obscure. Moreover, how autophagy induction (instead of suppression) affects IBAV infection has not yet been addressed.

Therefore, we investigated the relationship between IBAV infection and mTORC1, the most important signaling complex for autophagy regulation. We utilized amino acid starvation and mTOR inhibitors to examine the effect of mTORC1 suppression on IBAV infection. We found that amino acid starvation for $1 \mathrm{~h}$ immediately after IBAV infection significantly enhanced IBAV replication, suggesting that the very early stages of infection (0-1 hours post infection, hpi) are critical for the starvation-induced enhancement of IBAV replication. Unexpectedly, mTOR inhibitors used in this study (Torin 1 and rapamycin) did not increase IBAV replication, although these inhibitors sustained the inhibition of mTORC1 for longer time periods, suggesting that mTORC1 inhibition itself does not affect IBAV replication. We further showed that endosomal acidification is strongly induced by amino acid starvation but not by mTOR inhibitors, and that suppression of endosomal acidification by bafilomycin A1 abolished enhancement of IBAV replication in amino acid-starved cells. Therefore, for IBAV and related viruses such as BTV, a drug that suppresses endosomal acidification may be an effective method for suppressing viral replication. Our findings also indicate that amino acid starvation may provide an effective research tool for increasing the virus recovery rate of IBAV and related viruses.

\section{Materials \& Methods}




\subsection{Cells and viruses}

HmLu-1 (hamster lung) cells were maintained in Dulbecco's modified Eagle medium (DMEM; Wako) and supplemented with 10\% fetal bovine serum (FBS; Gibco), penicillin (100 U/ml), streptomycin $(100 \mu \mathrm{g} / \mathrm{ml})$, and L-glutamine $(0.29 \mathrm{mg} / \mathrm{ml})$.

IBAV was obtained from the National Institute of Animal Health, Japan, and propagated in HmLu-1 cells. Virus titer was determined by a plaque assay using HmLu1 cells with results reported as plaque forming units (pfu).

\subsection{Reagents}

Bafilomycin $\mathrm{A} 1$ (baf $\mathrm{A} 1$ ), a specific inhibitor of vacuolar-type $\mathrm{H}^{+}$-ATPase, was purchased from Adipogen. Torin 1, an mTORC1/2 inhibitor, was purchased from Tocris. Rapamycin, an mTORC1 inhibitor, and cOmplete protease inhibitor cocktail were purchased from Sigma-Aldrich. DMEM without amino acids was purchased from Wako. LysoTracker, a fluorescent indicator of cellular acidic fractions, was purchased from Thermo Fisher Scientific.

\subsection{IBAV infection in HmLu-1 cells}

HmLu-1 cells prepared in 6-well plates were chilled on ice for 5 min. After removing the media, cells were infected with IBAV at a multiplicity of infection (MOI) of 3 for $1 \mathrm{~h}$ at $4{ }^{\circ} \mathrm{C}$ in DMEM. After washing with PBS(-), cells were further incubated for $1 \mathrm{~h}$ in a $\mathrm{CO}_{2}$ incubator $\left(37^{\circ} \mathrm{C}, 5 \% \mathrm{CO}_{2}\right)$ in media containing drugs as described in each figure (figures $1,3,4$, and 5). The $\mathrm{pH}$ of the media was adjusted to approximately 7.4. After this 1-h treatment, the media were replaced with DMEM containing 10\% FBS. Cells were further incubated and the culture supernatants and cells were harvested at the indicated time points.

\subsection{Quantification of IBAV replication in $\mathrm{HmLu}-1$ cells}

The number of infectious virus particles in the supernatant and cell lysate was determined by the plaque assay. The supernatant and the cell lysate were harvested together in a microcentrifuge tube and sonicated for $5 \mathrm{~min}$ to extract IBAV from the cells. After centrifugation at $3,000 \mathrm{rpm}(600 \times \mathrm{g})$ for $5 \mathrm{~min}$, the supernatant was subjected to the plaque assay. $\mathrm{HmLu}-1$ cells were prepared in 6-well plates and incubated with appropriate dilutions of virus-containing samples for $2 \mathrm{~h}$. After incubation, the virus was removed and DMEM containing $5 \%$ FBS and $0.75 \%$ agar was overlaid. Plates were then 
incubated for 4 days and then the cells were fixed and stained with staining solution ( $0.1 \%$ crystal violet in $10 \%$ buffered formalin and $20 \%$ methanol). Plaques were counted and the total virus amount in each sample was calculated.

2.5. Quantification of mTORC1 and autophagy activities in HmLu-1 cells infected with IBAV

HmLu-1 cells were infected with IBAV using the method described in section 2.3. Infected cells were harvested at the indicated time points as follows: after infection, the medium was removed, cells were then washed with PBS, lysed in cold cell lysis buffer (20 mM Tris- $\mathrm{HCl} \mathrm{pH}$ 7.5, $150 \mathrm{mM} \mathrm{NaCl}, 1 \mathrm{mM}$ EDTA, $1 \mathrm{mM}$ EGTA, $2.5 \mathrm{mM}$ pyrophosphate, $1 \mathrm{mM} \beta$-glycerophosphate, $1 \mathrm{mM}$ orthovanadate, $1 \%$ Triton $\mathrm{X}-100$, and the protease inhibitor cocktail, cOmplete), and incubated on ice for $5 \mathrm{~min}$. The lysates were then centrifuged at $14,000 \mathrm{rpm}(18,000 \times \mathrm{g})$ for $10 \mathrm{~min}$ at $4{ }^{\circ} \mathrm{C}$, and the supernatant was collected and mixed with $6 \times$ sample buffer (12\% SDS, $45 \%$ glycerol, $375 \mathrm{mM}$ Tris $\mathrm{pH} 6.8,0.03 \%$ bromophenol blue, and 0.6 M DTT). Samples were boiled for 5 min and stored at $-20{ }^{\circ} \mathrm{C}$. The activity of mTORC1 and autophagy in IBAV-infected cells was analyzed by western blot analysis.

2.6. Quantification of autophagy induced by mTOR inhibitors and amino acid starvation $\mathrm{HmLu}-1$ cells prepared in 6-well plates were incubated for $4 \mathrm{~h}$ at $37^{\circ} \mathrm{C}$ with $250 \mathrm{nM}$ Torin 1 or $10 \mathrm{nM}$ rapamycin in DMEM containing 10\% FBS, or with amino acid-free DMEM without FBS. Cells were washed with PBS, lysed in 6× SDS sample buffer, and boiled for $5 \mathrm{~min}$. Samples were stored at $-20^{\circ} \mathrm{C}$. The autophagy-inducing effect of $\mathrm{mTOR}$ inhibitors and amino acid starvation was analyzed by western blot analysis.

\subsection{SDS-PAGE and western blot analysis}

Cell lysate samples were subjected to sodium dodecyl sulfate-polyacrylamide gel electrophoresis (SDS-PAGE) using $10 \%$ or $15 \%$ gels. Proteins were transferred to a PVDF membrane (Immobilon-P, Merck Millipore), blocked in Tris-buffered saline (TBS) containing $0.1 \%$ Tween 20 and $2 \%$ skim milk, and then incubated with one of the following primary antibodies: mouse anti-IBAV antiserum (1:5000 dilution, prepared in our laboratory (Tsuruta et al., 2016)), mouse anti-IBAV NS3 antiserum (1:5000; prepared in our laboratory (Urata et al., 2014)) rabbit anti-LC3 (1:1000; MBL), rabbit anti-phosphoS6K (Thr389; 1:1000; Cell Signaling Technology), rabbit anti-total S6K (1:1000; Cell Signaling Technology), and mouse anti-alpha tubulin (1:10000; Sigma-Aldrich). The 
membrane was then incubated with anti-mouse $\lg \mathrm{G}(\mathrm{H}+\mathrm{L})$ conjugated with $\mathrm{HRP}$ (1:5000; Jackson Immunoresearch) or anti-rabbit IgG $(H+L)$ conjugated with HRP (1:5000; Jackson Immunoresearch) as secondary antibodies. After incubation with substrate solution (EzWestLumi plus, Atto), the chemiluminescent signal was detected by LuminoGraph I (Atto) and quantified by CS Analyzer 4 software (version 2.2.4; Atto).

\subsection{Fluorescence observation of HmLu-1 cells by confocal microscopy}

HmLu-1 cells were plated on glass coverslips prepared in 6-well plates. For the LysoTracker experiment to visualize acidic endosomes/lysosomes, cells were washed once with PBS and incubated for $1 \mathrm{~h}$ at $37^{\circ} \mathrm{C}$ in drug-containing medium. The medium was then replaced with DMEM containing 10\% FBS without drugs. LysoTracker Red at $100 \mathrm{nM}$ was added to the medium $30 \mathrm{~min}$ before fixation. Cells treated with LysoTracker were washed twice with PBS and then fixed with 4\% paraformaldehyde in PBS for 20 min at room temperature. Cells on coverslips were washed twice with PBS, rinsed with pure water, and then mounted onto microscope slides with ProLong Gold anti-fade reagent with DAPI (Life Technologies). Fluorescence signals were observed by a confocal microscope (LMS710; Zeiss).

2.9. Quantification of IBAV replication in amino acid-starved cells in the presence of bafilomycin A1.

HmLu-1 cells prepared in 6-well plates were pre-treated with DMEM containing $10 \%$ FBS and $5 \mathrm{nM}$ of the v-ATPase inhibitor, baf $\mathrm{A} 1$, for $30 \mathrm{~min}$ at $37^{\circ} \mathrm{C}$. After cells were chilled on ice for $5 \mathrm{~min}$, the media were removed and cells were washed with PBS. The cells were then infected with IBAV at an MOI of 3 for $1 \mathrm{~h}$ at $4{ }^{\circ} \mathrm{C}$ in DMEM. Cells were washed with PBS and further incubated for $1 \mathrm{~h}$ at $37^{\circ} \mathrm{C}$ with DMEM or with amino acidfree DMEM containing $5 \mathrm{nM}$ baf $\mathrm{A} 1$ without FBS. After incubation, the media were replaced with DMEM containing $10 \%$ FBS. Cells were further incubated for $24 \mathrm{~h}$ and then the culture supernatant and cells were collected together. The supernatants and cells were subjected to the plaque assay as described in section 2.4 .

\subsection{Quantification of $m T O R$ inhibitors and amino acid starvation cytotoxicities}

Cytotoxicities of mTOR inhibitors and amino acid starvation were measured by the cell counting kit-8 (CCK-8; Dojindo). HmLu-1 cells plated in 96-well multiwell plates were chilled on ice for $5 \mathrm{~min}$. The medium was removed, and cells were incubated for $1 \mathrm{~h}$ at $4{ }^{\circ} \mathrm{C}$ in DMEM without IBAV infection. Cells were washed once with PBS and further 
incubated for $1 \mathrm{~h}$ at $37^{\circ} \mathrm{C}$ in medium containing various concentrations of inhibitors or in amino acid-free DMEM. Media were then replaced with DMEM containing 10\% FBS and after 11 or $23 \mathrm{~h}$ incubation, $10 \mu \mathrm{L}$ of the CCK-8 reagent was added to each well and incubated at $37^{\circ} \mathrm{C}$. After $1 \mathrm{~h}$, the absorbance at $450 \mathrm{~nm}$ was measured with the iMark microplate reader (Bio-Rad).

\subsection{Statistical analysis}

Statistical significance was analyzed using GraphPad Prism 7 (GraphPad Software; version 7.0a) to run unpaired t-tests (Figures $1 \mathrm{~A}$ and 3 ) and one-way analysis of variance (ANOVA) followed by Dunnett's test (Figures 1B, 1D, 4).

\section{Results}

\subsection{IBAV replication is enhanced by amino acid starvation}

It has been well established that autophagy is induced by amino acid starvation (Mizushima et al., 2011). In the absence of amino acids in the extracellular fluid, the activity of $\mathrm{mTORC} 1$ - a key negative regulator of autophagy -is strongly suppressed, resulting in the induction of autophagy. Therefore, we first examined the effect of amino acid starvation on IBAV replication following the protocol outlined in supplemental figure 1. IBAV were attached to hamster lung ( $\mathrm{HmLu}-1)$ cells for $1 \mathrm{~h}$ at $4{ }^{\circ} \mathrm{C}$, followed by treatment with amino acid-free medium. Note that amino acid starvation was limited to the early stages of post-IBAV infection (i.e., 0-1 hpi). After the 1-h starvation period, the medium was replaced with growth medium and incubated at $37^{\circ} \mathrm{C}$ until the supernatant and cells were harvested. The supernatant and cells were harvested together and then the number of infectious virus particles was determined by a plaque assay. We found that the IBAV titer of amino acid-starved cells was higher than that of the control cells at 12, 24, and 36 hpi (Fig. 1A). Based on this experiment, we decided to measure IBAV replication at 24 hpi hereafter, as this is around the earliest time point at which we can yield sufficiently large numbers of progeny viruses for reliable data acquisition. We attempted to examine progeny viruses as early as possible to examine the direct, and not secondary, effects of amino acid starvation.

3.2. Effect of mTOR inhibitors on IBAV replication is different from that of amino acid starvation 
Next, we examined if the mTOR inhibitors, Torin 1 and rapamycin, increase IBAV replication in a similar manner to amino acid starvation. Torin 1 inhibits both mTORC1 and mTORC2, and rapamycin inhibits only mTORC1 (Thoreen et al., 2009). We first performed an MTT assay to examine the cytotoxicity of these treatments on non-infected $\mathrm{HmLu}-1$ cells. This assay measures cellular metabolic activity and is commonly used as a readout of cell viability. Based on the MTT assay results, concentrations of Torin 1 and rapamycin to be used in the following experiments were determined as $250 \mathrm{nM}$ and 10 $\mathrm{nM}$, respectively, as these concentrations did not reduce total cellular metabolic activity. In addition, 1-h treatments with amino acid-free DMEM did not appear to reduce metabolic activity (Supplemental Fig. 2).

Next, the effect of the mTOR inhibitors on IBAV replication was tested as described for the amino acid starvation protocol indicated in supplemental figure 1 . HmLu-1 cells were treated with the mTOR inhibitors ( $250 \mathrm{nM}$ Torin 1 or $10 \mathrm{nM}$ rapamycin) or amino acid-free DMEM for $1 \mathrm{~h}$ immediately after infection. In contrast to the increased virus titers observed for amino acid-starved cells, virus titers of cells treated with Torin 1 or rapamycin were comparable to that of the control (Fig. 1B). In addition, we examined the expression levels of the IBAV proteins VP5 and NS3 by western blot analysis. Similar to the plaque assay results (Fig. 1A and B), the expression levels of these IBAV proteins were significantly enhanced by amino acid starvation but not by Torin 1 and rapamycin (Fig. 1C and D). These results indicate that unlike amino acid starvation, $250 \mathrm{nM}$ of Torin 1 and $10 \mathrm{nM}$ of rapamycin do not enhance IBAV replication.

3.3. Confirmation of autophagy induction by mTOR inhibitors and amino acid starvation Since the mTOR inhibitors did not affect IBAV replication, we suspected that the inhibitors were not effective in HmLu-1 cells even though the concentrations applied (250 $\mathrm{nM}$ Torin 1 and $10 \mathrm{nM}$ rapamycin) are within the commonly used range (Thoreen et al., 2009). Therefore, we attempted to confirm if the mTOR inhibitors effectively induced autophagy in HmLu-1 cells. To this end, uninfected HmLu-1 cells were treated with mTOR inhibitors and amino acid-free medium for $4 \mathrm{~h}$ with or without the lysosomal inhibitor, baf A1. Next, the expression of LC3-an indicator of autophagy-was analyzed by western blotting. LC3 is a protein that is covalently conjugated to autophagosomal membranes, and western blot analysis can differentiate between unconjugated cytosolic LC3 (LC3-I) and autophagosomal membrane-conjugated LC3 (LC3-II). Upon autophagy induction, LC3-I is converted to LC3-II, but is also degraded by autophagy. Therefore, to quantify autophagic activity, LC3-II turnover was calculated by [LC3-II with baf A1] - 
[LC3-II without baf A1] (Mizushima and Yoshimori, 2007). As shown in figure 2, $250 \mathrm{nM}$ Torin 1 and $10 \mathrm{nM}$ rapamycin enhanced autophagy. Altogether, it was confirmed that the mTOR inhibitors did not enhance IBAV replication even though they induce autophagy in $\mathrm{HmLu}-1$ cells. This suggests that induction of autophagy itself may not be sufficient to enhance IBAV replication.

\subsection{Examination of the effect of fetal bovine serum on IBAV replication}

Fetal bovine serum (FBS) contains amino acids and growth factors that activate mTORC1 and may possibly influence IBAV replication. Therefore, we examined if IBAV titer under an amino acid-starved condition is affected by FBS. HmLu-1 cells were treated with amino acid-free medium with or without $10 \%$ FBS for $1 \mathrm{~h}$ after infection. Then, the IBAV titer was determined by a plaque assay at $24 \mathrm{hpi}$. IBAV titer increased after amino acid starvation regardless of FBS supplementation, although IBAV titer without FBS was slightly higher than that with FBS (Supplemental Fig. 3). This result suggests that a small amount of amino acids in FBS may slightly impede the enhanced IBAV replication of amino acid-starved cells, but not enough to cause significant differences. Nevertheless, we decided to use FBS-free media for the 1-h post-infection treatments of further experiments to eliminate the effect caused by FBS amino acids.

\subsection{Effect of IBAV infection on mTORC1 activity and LC3-II accumulation.}

We examined how IBAV infection together with the mTOR inhibitors or amino acid starvation affects mTORC1 and autophagy activities. First, we focused on the effect of IBAV infection. The activity of mTORC1 was monitored by the phosphorylation of S6K at Thr389 that is directly phosphorylated by mTORC1. The difference in mTORC1 activity between non-infected and IBAV-infected cells was not apparent at least until $24 \mathrm{hpi}$ (Fig. $3 A$ and $B$ ). This result suggests that IBAV replication inside the host cell does not affect mTORC1 activity at early stages of IBAV infection (0-24 hpi). mTORC1 activity decreased at $48 \mathrm{hpi}$ in both uninfected and IBAV-infected cells, possibly due to the depletion of mTORC1 stimulatory signals (e.g. amino acids) in the medium during the incubation period. In addition, mTORC1 activity tended to be lower in IBAV-infected cells than in uninfected cells at $48 \mathrm{hpi}$, but statistical significance was not observed. On the other hand, the amount of LC3-II increased at 48 hpi in IBAV-infected cells (Fig. 3C and D). This accumulation of LC3-II was not detected at earlier time points up to $24 \mathrm{hpi}$, suggesting that LC3-II accumulation-indicative of enhanced autophagosome formation 
and/or suppressed autophagosome digestion-is caused by late events during IBAV replication.

3.6. mTORC1 activity and LC3-II accumulation in IBAV-infected, mTORC1-suppressed cells

Since IBAV titer increased after amino acid starvation but not by mTOR inhibitors, we examined if there were differences in mTORC1 activity and autophagy between amino acid-starved cells and cells treated with the inhibitors. In this experiment, cells were infected with IBAV and then treated with mTOR inhibitors or amino acid-free medium for $1 \mathrm{~h}$, and then the cells were harvested for western blot analysis at multiple time points between 0 and $48 \mathrm{hpi}$. As shown in figure $4 A$ and $B$, the control cells that were treated with FBS-free medium for $1 \mathrm{~h}$ after IBAV infection showed relatively stable mTORC1 activity. In amino acid-starved cells, there was an efficient decrease in phospho-S6K levels at $1 \mathrm{hpi}$ that were then recovered to normal levels immediately after the medium was replaced with DMEM containing $10 \%$ FBS (1.5 hpi). This suggests that mTORC1 suppression by amino acid starvation is reversible and that addition of amino acids can quickly reverse mTORC1 suppression. In contrast, phospho-S6K in cells treated with mTOR inhibitors was maintained at low levels even after $1.5 \mathrm{hpi}$. This shows that mTOR inhibitors remain effective even after the medium is replaced with an inhibitorfree medium at $1 \mathrm{hpi}$. On the other hand, quantification of LC3-II indicated that LC3-II accumulated at $48 \mathrm{hpi}$ in all conditions due to IBAV infection (Fig. 4C and D). At earlier time points (1-24 hpi), LC3-II tended to be accumulated in amino acid-starved, Torin 1treated, and rapamycin-treated cells compared to the control cells; however, no significant statistical differences were observed between the control group and each treatment group for all time points (Fig. 4C and D). Altogether, the results indicate that amino acid starvation, but not mTOR inhibitors, enhances IBAV replication (Fig. 1 and Supplemental Fig. 3) even though suppression of mTORC1 is less sustainable in amino acid-starved cells than in mTOR inhibitor-treated cells (Fig. 4A and B) and LC3-II accumulation is not significantly different between the control, amino acid-starved, and mTOR inhibitor-treated groups (Fig. 4C and D). This suggests that forced autophagy induction through mTORC1 suppression does not contribute to the enhancement of IBAV replication.

3.7. Acidification of endosomes is promoted by amino acid starvation. 
The relationship between IBAV replication and mTORC1 suppression/autophagy was not established in our experimental conditions; therefore, we suspected that there are other mechanisms by which amino acid starvation enhances IBAV replication. We recently showed that IBAV exploits the endocytic pathway to enter host cells, and that acidification within endosomes act as critical triggers for IBAV infection (Tsuruta et al., 2016). In addition, it was reported that acidification of endosomes/lysosomes is enhanced by nutrient starvation and mTOR inhibitors (Zhou et al., 2013). Consistent with enhanced acidification, lysosomal digestion of endocytosed proteins was also enhanced by mTOR inhibition (Palm et al., 2015). Based on these previous studies, we hypothesized that endosomal acidification is the key to IBAV replication enhancement by amino acid starvation. To test this hypothesis, we stained uninfected cells with the fluorescent LysoTracker dye (Fig. 5A) to examine if the staining is specifically affected by amino acid starvation, as the dye accumulates in acidified cellular compartments. Indeed, cells starved of amino acids for $1 \mathrm{~h}$ exhibited a more intense staining of endosomal/lysosomal punctate structures. Treatment with $250 \mathrm{nM}$ Torin 1 for $1 \mathrm{~h}$ slightly increased LysoTracker staining although the enhancement was much weaker than after amino acid starvation. After 1-h treatment of amino acid starvation or by mTOR inhibitors, the medium was replaced by growth medium (DMEM containing 10\% FBS). The cells were then incubated for an additional $2 \mathrm{~h}$ (total $3 \mathrm{~h}$ ) or $5 \mathrm{~h}$ (total $6 \mathrm{~h}$ ) in growth medium and the staining intensity became comparable to that of the control, indicating that enhancement of endosomal/lysosomal acidification by amino acid starvation was reversible (Fig. 5A). This is consistent with the reversible suppression of mTORC1 by amino acid starvation (Fig. 4). These findings indicate that enhancement of IBAV replication is possibly due to enhanced acidification of endosomes, both of which were specific to amino acid starvation and were not detected in cells treated with mTOR inhibitors.

3.8. Enhancement of IBAV replication by amino acid starvation is suppressed by baf A1.

Due to the possibility of IBAV replication enhancement by increased endosome acidification, we examined if IBAV replication in amino acid starved cells is suppressed by the $v$-ATPase inhibitor, baf A1, that inhibits acidification of endosomes and lysosomes. $\mathrm{HmLu}-1$ cells were pre-treated with baf $\mathrm{A} 1$ for $30 \mathrm{~min}$, incubated with IBAV for $1 \mathrm{~h}$ at $4{ }^{\circ} \mathrm{C}$, starved of amino acids in the presence of baf $A 1$ for $1 \mathrm{~h}$, and then harvested for plaque assays at $24 \mathrm{hpi}$. As shown in figure 5B, enhanced IBAV replication in amino acid-starved cells was almost completely suppressed by baf $A 1$. This suggests that endosome 
acidification is a critical event for IBAV replication enhancement in amino acid-starved cells.

\section{Discussion}

In this study, we investigated the effects of amino acid starvation and mTOR inhibitors on IBAV replication. Amino acid starvation and mTOR inhibitors result in the suppression of mTORC1, which in turn leads to an induction of autophagy. It was previously suggested that IBAV replication is suppressed by pharmacological and genetic inactivation of autophagy (Shai et al., 2013); in contrast to this previous study that focused on the effects of autophagy inhibition, we examined autophagy-inducing conditions.

We found that amino acid starvation for 0-1 hpi increased IBAV titer (Fig. 1). Unexpectedly, the mTOR inhibitors Torin 1 and rapamycin did not increase IBAV titer, (Fig. 1B) even though they suppressed mTORC1 activity at 1-24 hpi more strongly than amino acid starvation (Fig. 4A and B). Consistent with these findings, an increase in LC3II accumulation - which reflects autophagy activity - was induced by the inhibitors as well as by amino acid starvation (Fig. 2, 4C and D). These results suggest that autophagy induction and mTORC1 suppression are not sufficient to enhance IBAV replication.

Since we could not confirm the involvement of autophagy in the enhanced IBAV replication in amino acid-starved cells, we sought to identify other critical factors. Previous studies reported that lysosomal acidification is enhanced by amino acid starvation and mTOR inhibitors (Zhou et al., 2013) and that degradation of endocytosed proteins is activated by Torin 1 (Palm et al., 2015). Mechanistically, amino acid starvation enhances the assembly of the lysosomal proton pump, v-ATPase complex (Stransky and Forgac, 2015), and increases transcription factor EB (TFEB)-dependent transcription of lysosomal genes (Zhou et al., 2013). Notably, it was also reported that IBAV replication is enhanced by the acidification of host cell endosomes (Tsuruta et al., 2016). Based on these previous studies, we suspected that endosomal acidification may be a critical factor for IBAV replication enhancement in amino acid-starved cells. This hypothesis is supported by our finding that endosome acidification was strongly enhanced in amino acid-starved cells but was not apparent in Torin 1- or rapamycin-treated cells (Fig. 5A). Furthermore, enhanced IBAV replication by amino acid starvation was almost completely blocked by bafilomycin $A 1$, an inhibitor of the v-ATPase proton pump that leads to 
inhibition of endosome/lysosome acidification (Fig. 5B). The study by Zhou et al. showed that Torin 1 enhanced endosome acidification; conversely, our data indicate that Torin 1 has no apparent effect (Zhou et al., 2013). This discrepancy is possibly due to the difference in Torin 1 concentrations (1000 nM versus the $250 \mathrm{nM}$ used in this study), treatment lengths ( $3 \mathrm{~h}$ versus $1 \mathrm{~h}$ in this study), and cell lines used (mouse embryonic fibroblast and HeLa cells versus HmLu-1 cells in this study).

Consistent with a previous study by Shai et al. (Shai et al., 2013), we observed the accumulation of LC3-II in IBAV-infected cells at 48 hpi (Fig. 3C and D). LC3-II is the autophagosome-attached form of LC3, and the amount of LC3-II reflects the balance of autophagosome generation and destruction (Mizushima and Yoshimori, 2007). Therefore, the observed accumulation of LC3-II in IBAV-infected cells can result from increased autophagosome generation and/or decreased autophagosome digestion. Since we observed a slight decrease in mTORC1 activity in IBAV-infected cells at $48 \mathrm{hpi}$ (Fig. 3A and $B$ ), this may contribute to autophagosome generation induced in IBAVinfected cells. Alternatively, it is possible that the lysosomal digestion of autophagosomes is decreased in IBAV-infected cells, considering that IBAV enters the host cell via endocytosis. After IBAV is endocytosed, it ruptures the endosomal membrane to access the cytoplasm (Tsuruta et al., 2016). This endosomal rupture is more likely to occur in matured endosomes, as their enhanced acidification acts as a trigger for IBAV replication (Tsuruta et al., 2016). Since matured endosomes (late endosomes) play an important role in lysosomal maturation (Saftig and Klumperman, 2009), damage to late endosomes is likely to impair lysosomal function, resulting in decreased lysosomal LC3-II digestion.

Taken together, in contrast to the previous study that suggested the requirement of autophagy for optimal IBAV replication (Shai et al., 2013), our present study indicates that the forced induction of autophagy through mTORC1 suppression is not sufficient for the enhancement of IBAV replication. Instead, endosomal acidification induced by amino acid starvation, but not by mTOR inhibitors, appears to play a significant role in the enhanced IBAV replication.

\section{Conclusions}

Our findings revealed that the strong inactivation of mTORC1 induced by amino acid starvation during the early stages of infection (0-1 hpi) prominently increased endosome acidification, which in turn enhanced IBAV replication. Therefore, amino acid starvation (or strong mTORC1 inhibition) may also enhance the replication of other viruses that 
enter host cells via endocytosis and exploit endosomal acidification as a trigger to invade the cytoplasm. In the case of IBAV and other related viruses, inhibitors of endosomal acidification may be effective at suppressing viral replication. Furthermore, amino acid starvation may be employed as an effective research tool for isolation of IBAV and related viruses as it increases virus recovery rate.

\section{Conflict of interest}

The authors declare that they have no conflicts of interest regarding the contents of this study.

\section{Acknowledgements}

The authors thank Dr. Ken Maeda of the Joint Faculty of Veterinary Medicine Yamaguchi University for his valuable insights. The authors also thank the members of the Laboratory of Veterinary Hygiene for their cooperation in accomplishing this study. This work was supported by grants from JSPS KAKENHI (Grant Number 15K07745) to H.I., JSPS KAKENHI (Grant Number 15K18530), Funds for the Development of Human Resources in Science and Technology under MEXT through the Home for Innovative Researchers and Academic Knowledge Users (HIRAKU) consortium, the Ichiro Kanehara Foundation, and Yamaguchi University Foundation to S.S. 


\section{References}

Chi, P.I., Huang, W.R., Lai, I.H., Cheng, C.Y., Liu, H.J., 2013. The p17 nonstructural protein of avian reovirus triggers autophagy enhancing virus replication via activation of phosphatase and tensin deleted on chromosome 10 (PTEN) and AMPactivated protein kinase (AMPK), as well as dsRNA-dependent protein kinase (PKR)/elF2 $\alpha$ signaling pathways. J. Biol. Chem. 288, 3571-3584. https://doi.org/10.1074/jbc.M112.390245

Dreux, M., Chisari, F.V., 2010. Viruses and the autophagy machinery. Cell Cycle 9, 1295-1307. https://doi.org/10.4161/cc.9.7.11109

Gould, A.R., Hyatt, A.D., 1994. The orbivirus genus. Diversity, structure, replication and phylogenetic relationships. Comp. Immunol. Microbiol. Infect. Dis. 17, 163-188.

Gu, L., Musiienko, V., Bai, Z., Qin, A., Schneller, S.W., Li, Q., 2012. Novel virostatic agents against bluetongue virus. PloS One 7, e43341. https://doi.org/10.1371/journal.pone.0043341

Hirashima, Y., Kato, T., Yamakawa, M., Shirafuji, H., Okano, R., Yanase, T., 2015. Reemergence of Ibaraki disease in southern Japan in 2013. J. Vet. Med. Sci. 77, 1253-1259. https://doi.org/10.1292/jvms.15-0039

Inaba, U., 1975. Ibaraki disease and its relationship to bluetongue. Aust. Vet. J. $51,178-185$.

Jackson, W.T., 2015. Viruses and the autophagy pathway. Virology 479-480, 450-456. https://doi.org/10.1016/j.virol.2015.03.042

Lv, S., Xu, Q., Sun, E., Yang, T., Li, J., Feng, Y., Zhang, Q., Wang, H., Zhang, J., Wu, D., 2015. Autophagy Activated by Bluetongue Virus Infection Plays a Positive Role in Its Replication. Viruses 7, 4657-4675. https://doi.org/10.3390/v7082838

Meng, S., Jiang, K., Zhang, X., Zhang, M., Zhou, Z., Hu, M., Yang, R., Sun, C., $\mathrm{Wu}, \mathrm{Y} ., 2012$. Avian reovirus triggers autophagy in primary chicken fibroblast cells and Vero cells to promote virus production. Arch. Virol. 157, 661-668.

https://doi.org/10.1007/s00705-012-1226-x

Mizushima, N., Komatsu, M., 2011. Autophagy: renovation of cells and tissues. Cell 147, 728-741. https://doi.org/10.1016/j.cell.2011.10.026

Mizushima, N., Yoshimori, T., 2007. How to interpret LC3 immunoblotting. Autophagy 3, 542-545. 
Mizushima, N., Yoshimori, T., Ohsumi, Y., 2011. The role of atg proteins in autophagosome formation. Annu. Rev. Cell Dev. Biol. 27, 107-132.

https://doi.org/10.1146/annurev-cellbio-092910-154005

Palm, W., Park, Y., Wright, K., Pavlova, N.N., Tuveson, D.A., Thompson, C.B., 2015. The Utilization of Extracellular Proteins as Nutrients Is Suppressed by mTORC1. Cell 162, 259-270. https://doi.org/10.1016/j.cell.2015.06.017

Saftig, P., Klumperman, J., 2009. Lysosome biogenesis and lysosomal membrane proteins: trafficking meets function. Nat. Rev. Mol. Cell Biol. 10, 623-635.

https://doi.org/10.1038/nrm2745

Saxton, R.A., Sabatini, D.M., 2017. mTOR Signaling in Growth, Metabolism, and Disease. Cell 168, 960-976. https://doi.org/10.1016/j.cell.2017.02.004

Shai, B., Schmukler, E., Yaniv, R., Ziv, N., Horn, G., Bumbarov, V., Yadin, H., Smorodinsky, N.I., Bacharach, E., Pinkas-Kramarski, R., Ehrlich, M., 2013. Epizootic hemorrhagic disease virus induces and benefits from cell stress, autophagy, and apoptosis. J. Virol. 87, 13397-13408. https://doi.org/10.1128/JVI.02116-13

Stransky, L.A., Forgac, M., 2015. Amino Acid Availability Modulates Vacuolar H+ATPase Assembly. J. Biol. Chem. 290, 27360-27369.

https://doi.org/10.1074/jbc.M115.659128

Thoreen, C.C., Kang, S.A., Chang, J.W., Liu, Q., Zhang, J., Gao, Y., Reichling, L.J., Sim, T., Sabatini, D.M., Gray, N.S., 2009. An ATP-competitive mammalian target of rapamycin inhibitor reveals rapamycin-resistant functions of mTORC1. J. Biol. Chem. 284, 8023-8032. https://doi.org/10.1074/jbc.M900301200

Tsuruta, Y., Shibutani, S.T., Watanabe, R., Iwata, H., 2016. The requirement of environmental acidification for Ibaraki virus infection to host cells. J. Vet. Med. Sci. 78, 153-156. https://doi.org/10.1292/jvms.15-0222

Urata, M., Watanabe, R., Iwata, H., 2014. The host specific NS3 glycosylation pattern reflects the virulence of Ibaraki virus in different hosts. Virus Res. 181, 6-10. https://doi.org/10.1016/j.virusres.2013.12.027

Zhou, J., Tan, S.-H., Nicolas, V., Bauvy, C., Yang, N.-D., Zhang, J., Xue, Y., Codogno, P., Shen, H.-M., 2013. Activation of lysosomal function in the course of autophagy via mTORC1 suppression and autophagosome-lysosome fusion. Cell Res. 23, 508-523. https://doi.org/10.1038/cr.2013.11 


\section{Figure legends}

Figure 1. IBAV replication is enhanced by amino acid starvation.

(A) The experiments were performed as shown in supplemental figure 1. HmLu-1 cells were incubated with IBAV at a multiplicity of infection (MOI) of 3 for $1 \mathrm{~h}$ at $4{ }^{\circ} \mathrm{C}$, and then treated with DMEM (control) or DMEM without amino acids ((-) a.a.) for $1 \mathrm{~h}$ at $37^{\circ} \mathrm{C}$. After the 1-h treatment, the medium was replaced by growth medium (DMEM containing $10 \%$ FBS). Culture supernatant and cells were collected together at the time points indicated in the graph. Virus titers were determined by plaque forming assays. Each bar indicates mean \pm standard error of the mean (SEM) of three independent experiments. ${ }^{* *}, \mathrm{p}<0.01 ;{ }^{* * *}, \mathrm{p}<0.001$.

(B) HmLu-1 cells were infected with IBAV and treated for $1 \mathrm{~h}$ with DMEM (control), amino acid-free DMEM ((-) a.a.), DMEM containing $250 \mathrm{nM}$ Torin 1, or DMEM containing $10 \mathrm{nM}$ rapamycin. All media contained 10\% FBS. After incubation in growth medium for an additional $23 \mathrm{~h}$, the cells and supernatant were collected together at 24 hours post infection (hpi). Virus titers were determined by plaque forming assays. Each bar indicates mean \pm SEM of three independent experiments. ${ }^{* * *}, p<0.001$ compared to the DMEMtreated control group.

(C) HmLu-1 cells infected with IBAV were treated as in (B). At $24 \mathrm{hpi}$, the cells were collected and subjected to western blot analysis. Astarisk indicates non-specific signals. (D) Quantification of westarn blot data in (C). Note that quantification of VP5 was not performed due to the non-specific signals. Each bar indicates mean \pm SEM of three independent experiments. ${ }^{* * *}, \mathrm{p}<0.0001$ compared to the IBAV-infected, DMEMtreated control group.

Figure 2. Autophagy-inducing ability of mTOR inhibitors and amino acid starvation. HmLu-1 cells were incubated with DMEM (control), DMEM containing $250 \mathrm{nM}$ Torin 1, DMEM containing $10 \mathrm{nM}$ rapamycin, or amino acid-free DMEM ((-) a.a.) for $4 \mathrm{~h}$ at $37^{\circ} \mathrm{C}$. Each treatment was performed with or without $100 \mathrm{nM}$ bafilomycin A1 (baf A1). All media contained $10 \%$ FBS except for (-) a.a.. After collecting, the cells were subjected to (A) western blot analysis, and $(B)$ the protein expression was quantified by densitometry. Note that the difference in the amounts of LC3-II with and without bafilomycin A1 reflects autophagic activity. A representative data set from two independent experiments are shown. Each bar indicates mean \pm SEM of three independent experiments. 
Figure 3. IBAV infection results in LC3-II accumulation without affecting mTORC1 activity.

$\mathrm{HmLu}-1$ cells were infected with IBAV and then treated with DMEM without FBS for $1 \mathrm{~h}$ (0-1 hpi) as shown in supplemental figure 1 . The cells were collected at the indicated time points, $(A, C)$ subjected to western blot analysis, and $(B, D)$ the protein expression was quantified by densitometry. Phospho-S6K (Thr389) reflects the activity of mTORC1 and the amount of LC3-II reflects the balance of generation and degradation of the autophagosome. Each bar indicates mean \pm SEM of four independent experiments. ${ }^{* * * *}$, $\mathrm{p}<0.0001$.

Figure 4. Effects of mTOR inhibitors and amino acid starvation on the activity of mTORC1 and the accumulation of LC3-II.

HmLu-1 cells were infected with IBAV for $1 \mathrm{~h}$ and treated for $1 \mathrm{~h}(0-1 \mathrm{hpi})$ with DMEM, amino acid-free DMEM ((-) a.a.), DMEM containing $250 \mathrm{nM}$ Torin 1, or DMEM containing $10 \mathrm{nM}$ rapamycin as shown in supplemental figure 1 . The cells were collected at the indicated time points, $(A, C)$ subjected to western blot analysis, and $(B, D)$ the protein expression was quantified by densitometry. Each bar indicates mean \pm SEM of four independent experiments. ${ }^{*}, p<0.05 ;{ }^{* *}, p<0.01 ;{ }^{* * *}, p<0.001 ;{ }^{* * *}, p<0.0001$ compared to the DMEM-treated control group.

Figure 5. Amino acid starvation increases IBAV replication via enhanced endosomal acidification.

(A) $\mathrm{HmLu}-1$ cells were incubated with the indicated medium without FBS for $1 \mathrm{~h}$ at $37^{\circ} \mathrm{C}$ and then further incubated in growth medium (DMEM containing 10\% FBS) for an additional 2 or $5 \mathrm{~h}$ (a total of 3 or $6 \mathrm{~h}$ ). LysoTracker with a final concentration of $50 \mathrm{nM}$ was added to the medium 30 min before fixation.

(B) HmLu-1 cells were pre-treated with DMEM containing 10\% FBS and $5 \mathrm{nM}$ baf $\mathrm{A} 1$ for $30 \mathrm{~min}$ at $37^{\circ} \mathrm{C}$. After cells were infected with IBAV for $1 \mathrm{~h}$ at $4{ }^{\circ} \mathrm{C}$, the cells were incubated with DMEM or amino acid-free DMEM (with or without $5 \mathrm{nM}$ baf $\mathrm{A} 1$ ) for $1 \mathrm{~h}$ at $37^{\circ} \mathrm{C}$, and then further incubated for $23 \mathrm{~h}$ in growth medium. The culture supernatant and cells were collected together, and then virus titers were determined by plaque forming assays. Each bar indicates mean \pm SEM of three independent experiments. 
Figure 1

A

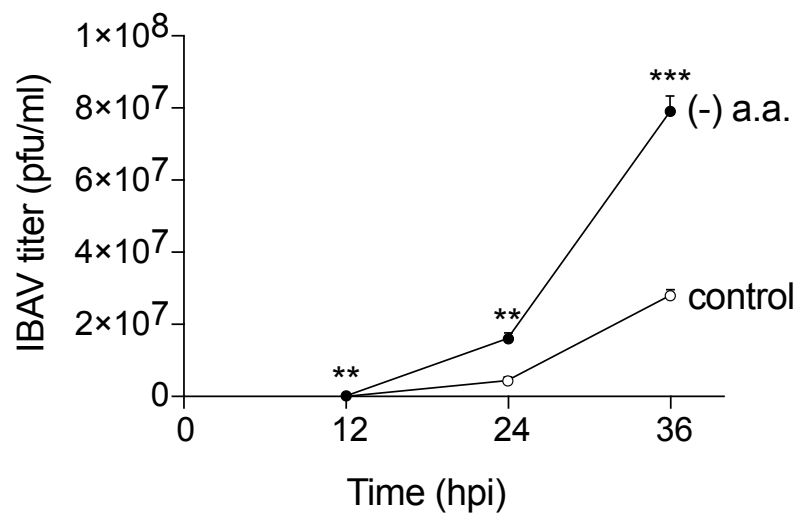

C

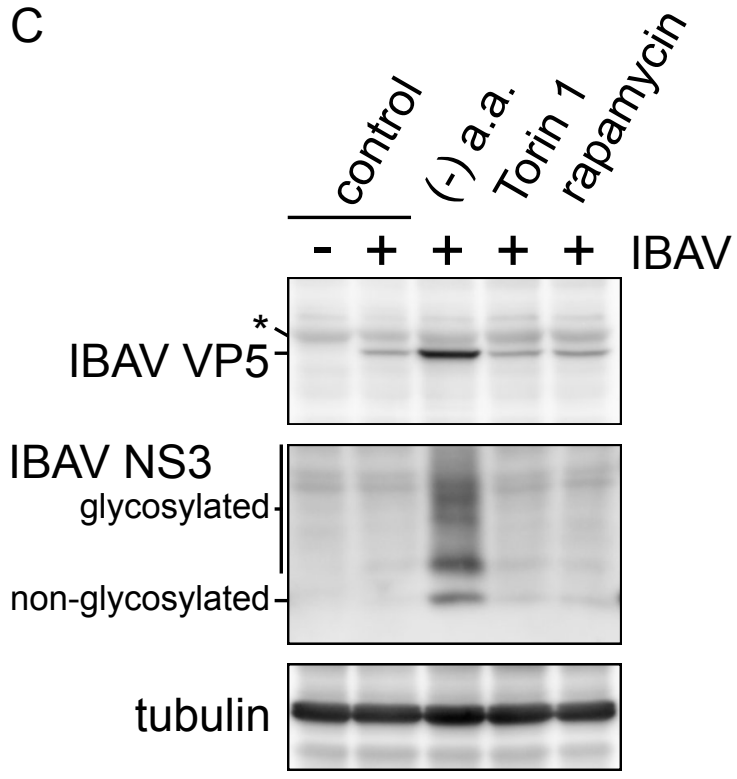

B

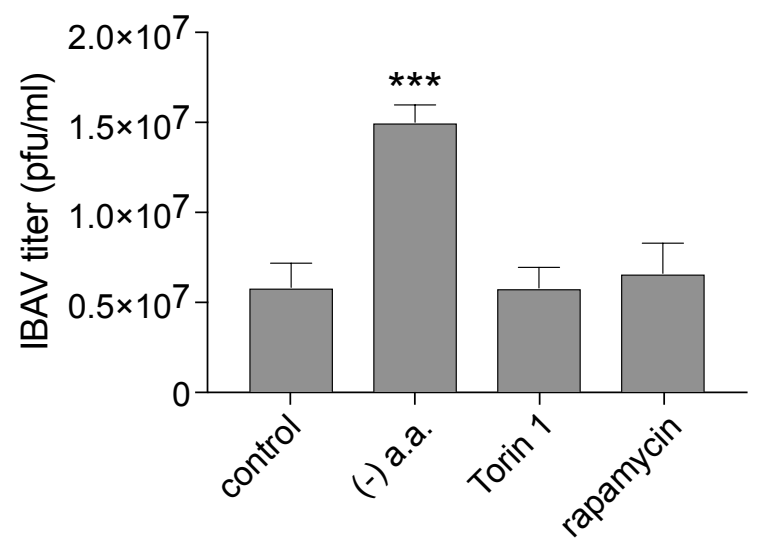

D

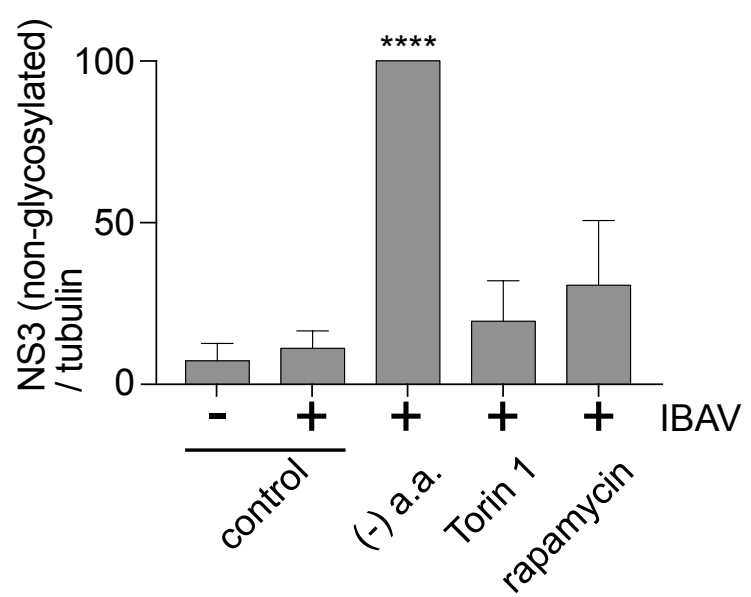


Figure 2
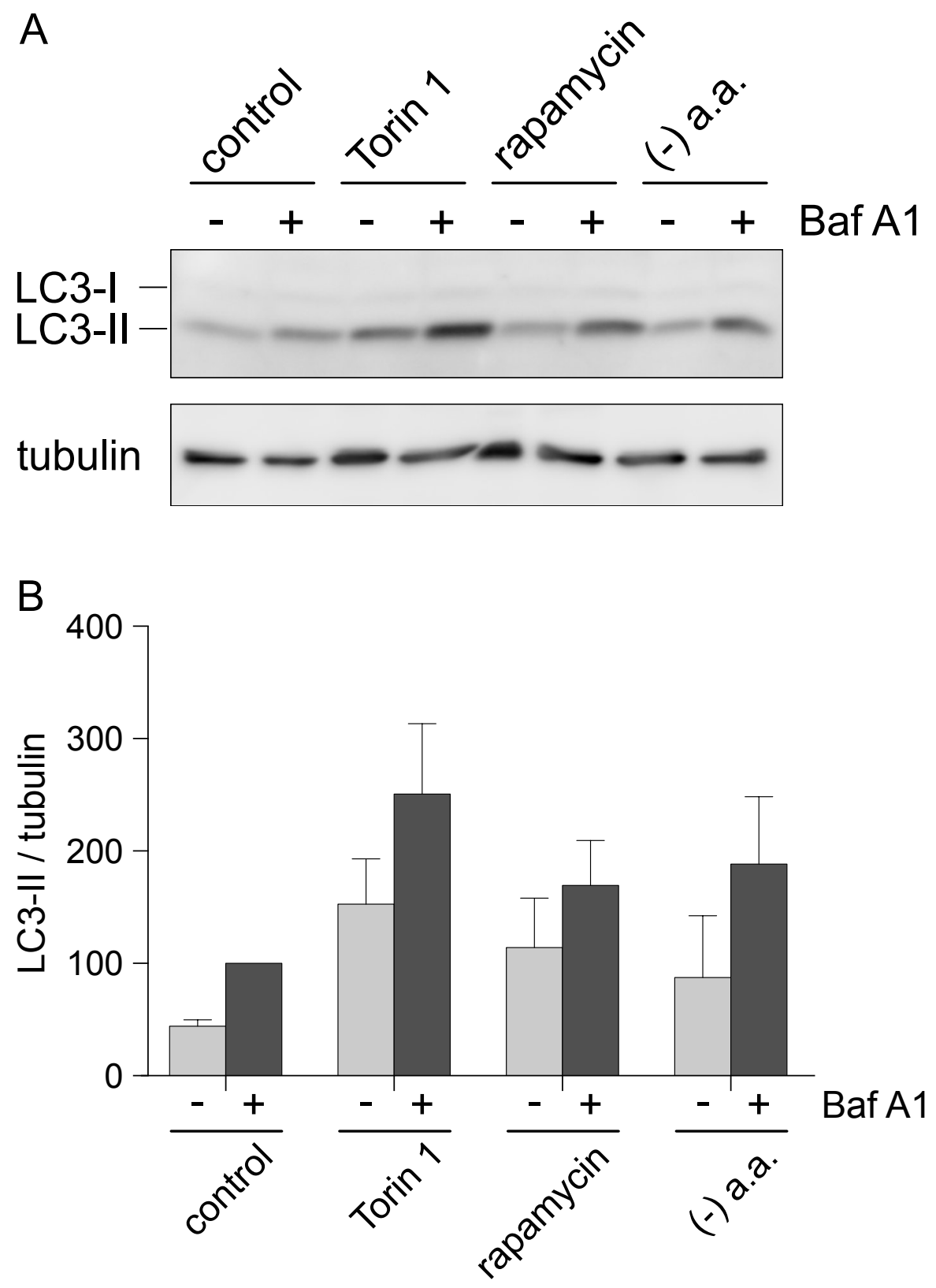
Figure 3

A

Time (hpi) 0
IBAV $\frac{1}{+} \frac{1.5}{+} \frac{24}{+} \frac{48}{+}$

phospho-S6K

total S6K

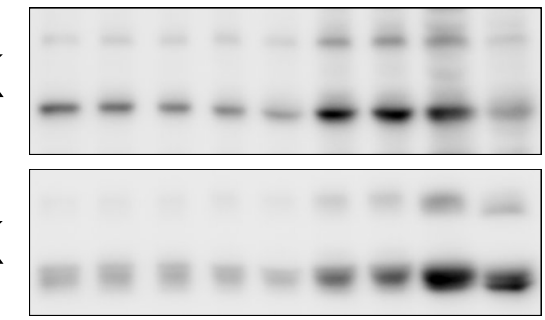

C

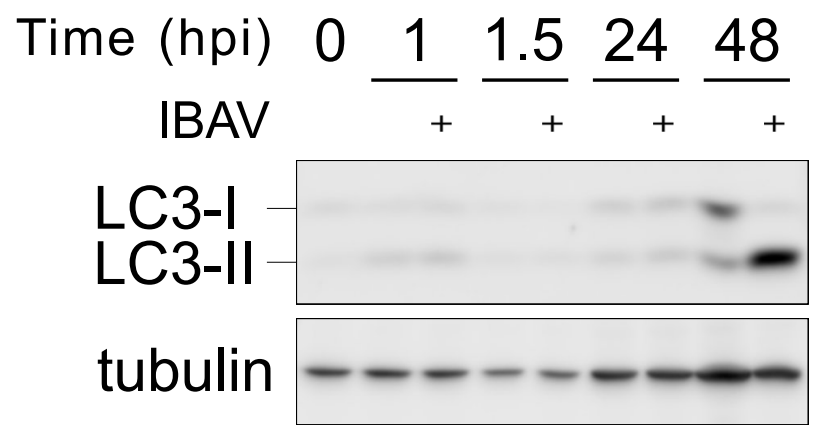

B
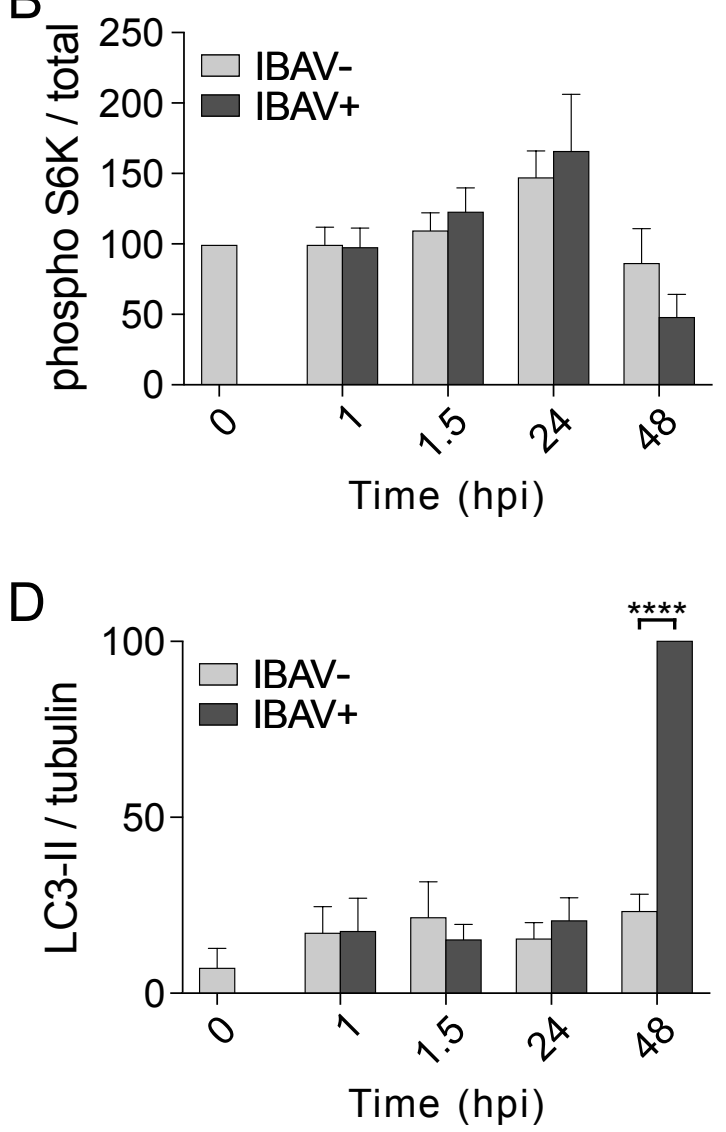
Figure 4

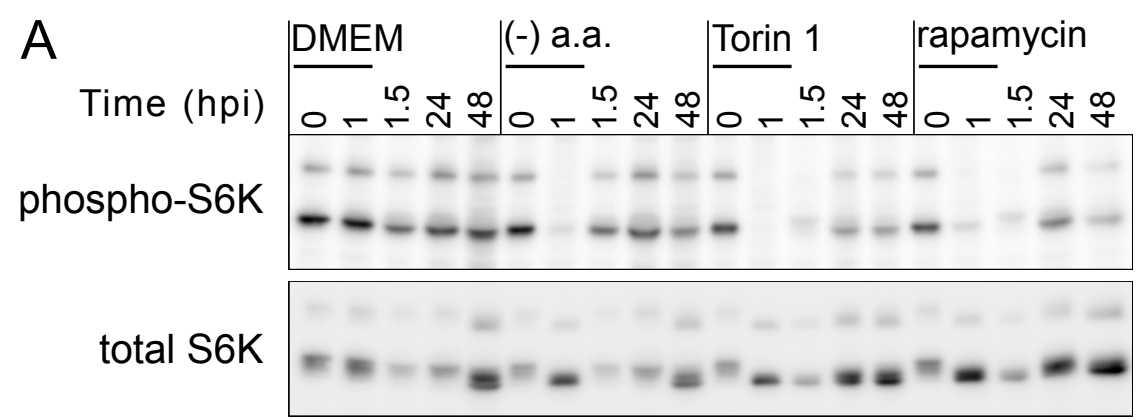

B

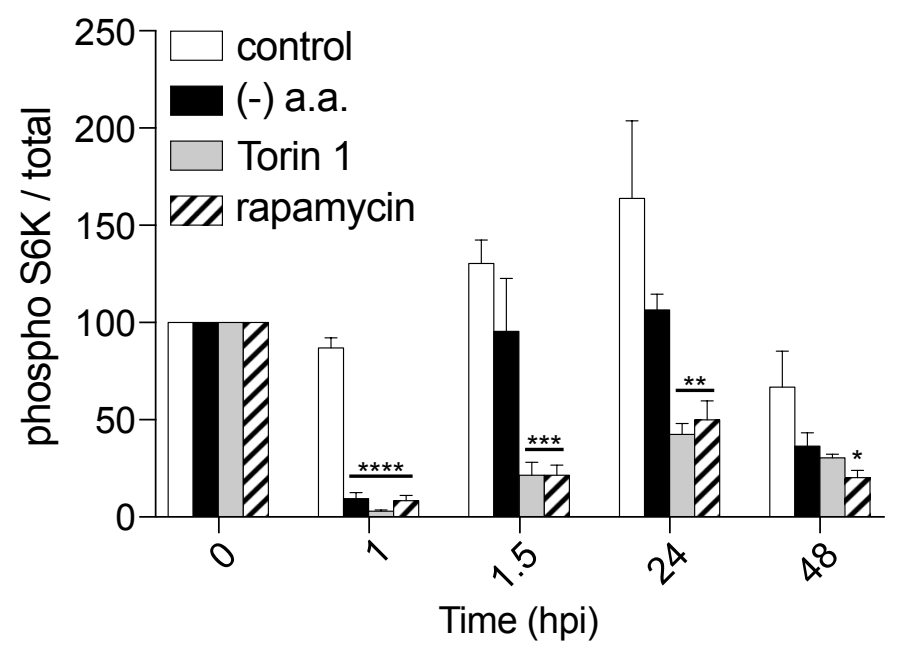

C

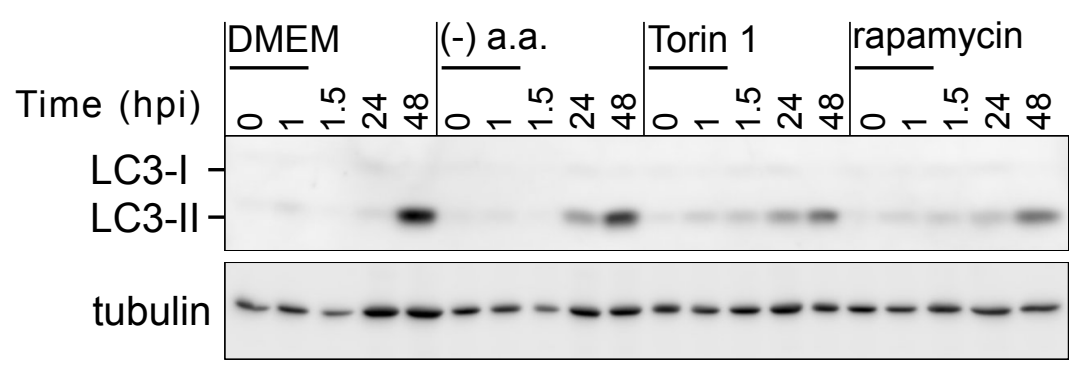

D

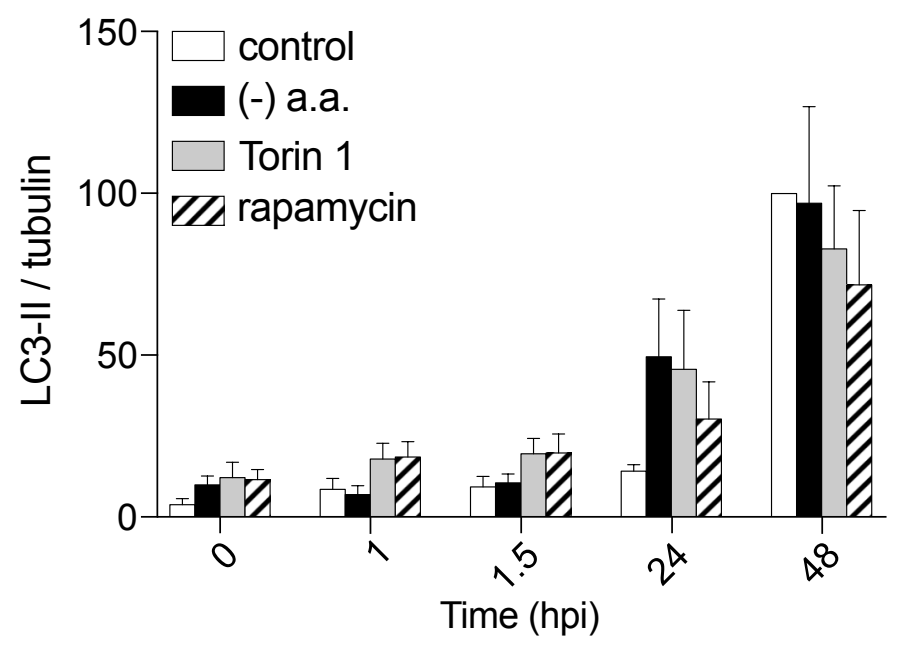


Figure 5 (Color figure)

A LysoTracker / DAPI

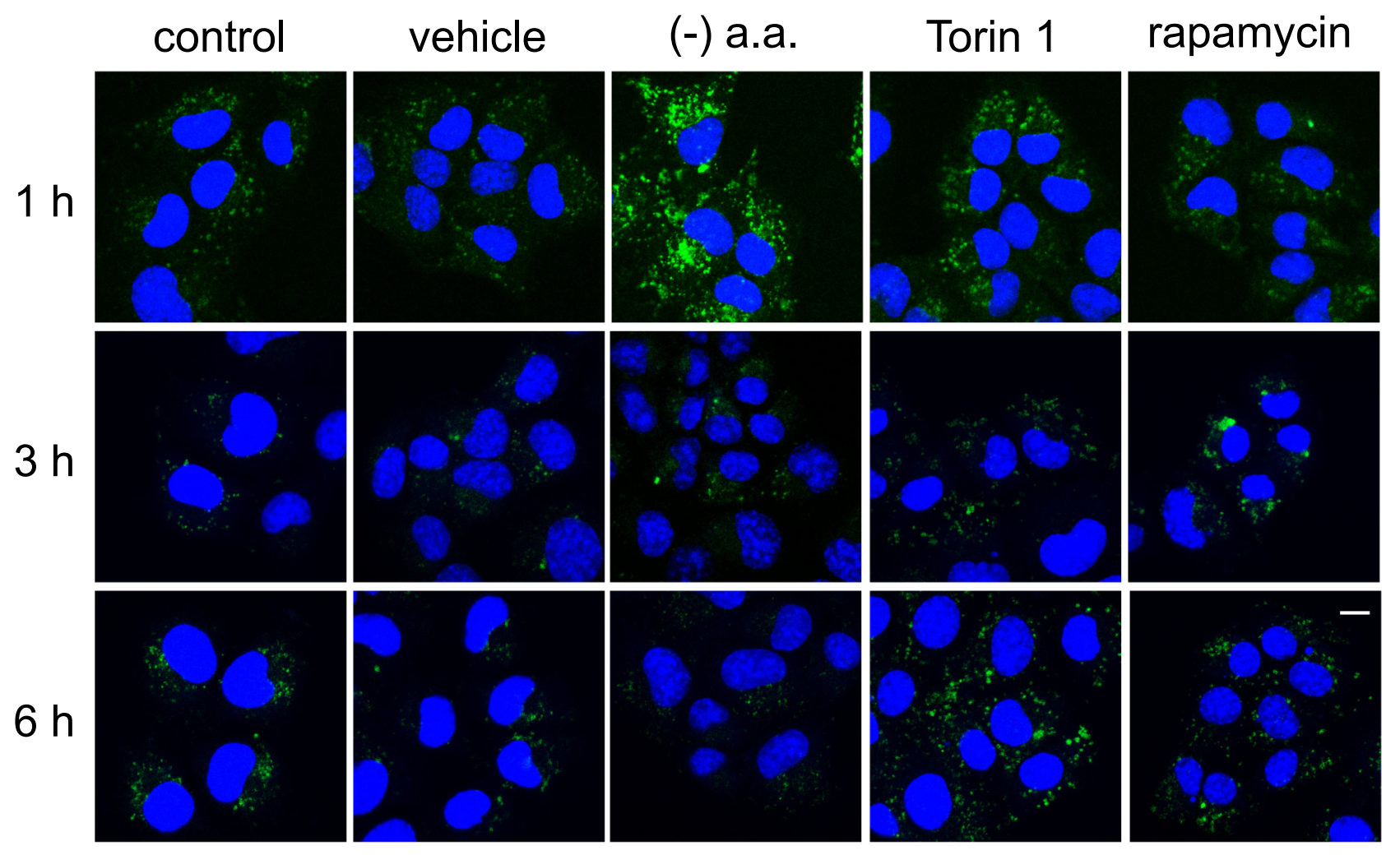

B

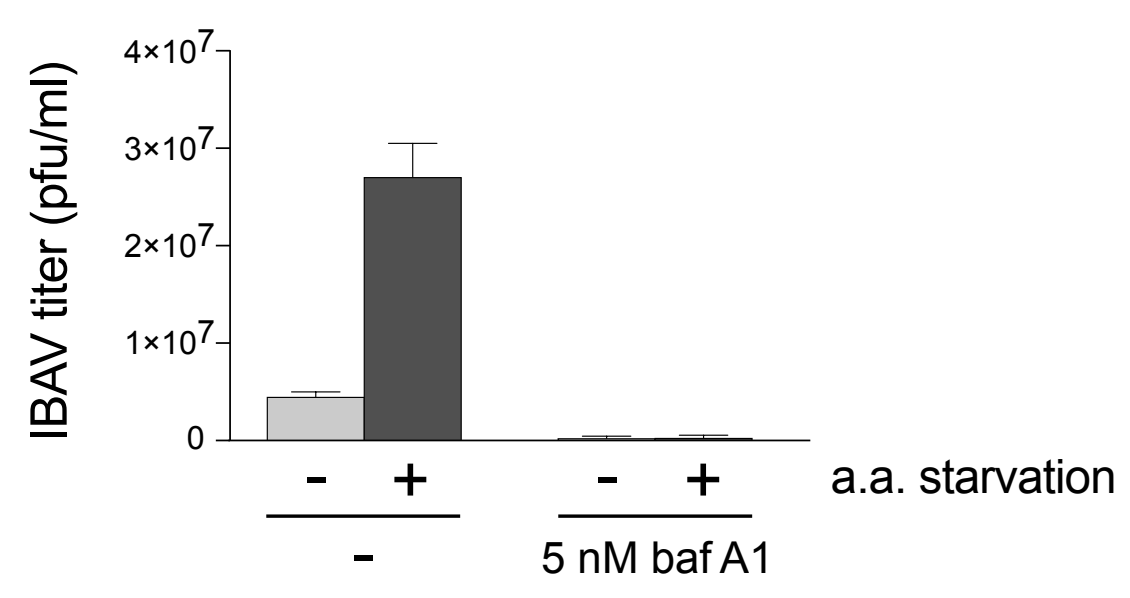

